\section{Kompass \\ Autoimmun}

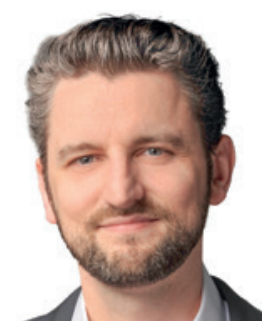

Jan Leipe

Sektion Rheumatologie, Medizinische Klinik V, Universitätsklinikum Mannheim, Mannheim, Deutschland

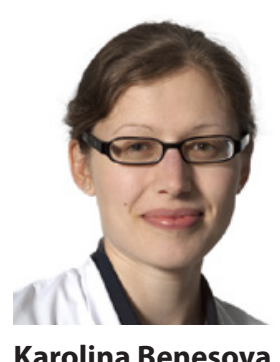

Sektion Rheumatologie, Innere Medizin V, Sektion Rheumatologie, Universitätsklinikum Heidelberg, Heidelberg, Deutschland

Onkologen, Rheumatologen und andere Spezialisten für muskuloskelettale Erkrankungen werden zunehmend mit Patienten konfrontiert, deren Symptome erstmals oder verstärkt unter einer Malignomtherapie mit Immun-Checkpoint-Inhibitoren (ICI) aufgetreten sind.

Die unspezifische T-Zellaktivierung unter ICI bedingt ein breites Spektrum an Nebenwirkungen, die auf entzündliche autoimmune oder autoinflammatorische Gewebeschäden zurückzuführen sind, sog. immunbedingte unerwünschte Ereignisse (engl.: immune related adverse events, irAE). Diese können potenziell jedes Organsystem betreffen; am häufigsten sind dermatologische, gastrointestinale und endokrine Manifestationen. Während in großen Zulassungsstudien diffuse muskuloskelettale Symptome (z.B. Arthralgien und Myalgien) und seltener $(\leq 1 \%)$ andere rheumatische irAEs (insbeson-

\title{
Immunbedingte unerwünschte Ereignisse unter Therapie mit Immun-Checkpoint-Inhibitoren
}

dere Arthritis und Myositis) beschrieben wurden, zeigt sich in der prospektiven Beobachtung jedoch, dass die wahre Inzidenz von inflammatorischen de novo (d.h. ohne vorbestehende entzündlich-rheumatische Erkrankung) rheumatischen irAEs eher bei 25\% liegt. Diese spiegeln das breite Spektrum bekannter rheumatischer Erkrankungen wider: Die häufigsten Manifestationen sind Arthritiden, Tendinitiden/Tenosynovialitiden, Enthesitiden, Polymyalgia rheumatica und Sarkoidose, aber auch Fallberichte über Vaskulitiden, Kollagenosen einschließlich Myositiden, systemischer Sklerose, Lupus erythematodes und Sicca-Syndrom sind publiziert. Aufgrund dessen, dass sich die rheumatischen irAEs vielfach bezüglich der klinischen Ausprägung z.T. deutlich von den klassischen Entitäten unterscheiden, wird bei rheumatischen irAEs oft der Zusatz «-ähnlich» (-like), z.B. «rheumatoide Arthritis-like», «Lupus-like», als Nomenklatur verwendet.

Neben den de novo rheumatischen irAEs kommt es bei Patienten mit vorbestehender entzündlich-rheumatischer Erkrankung zu einer Zunahme der Krankheitsaktivität ( 40\%) oder Auftreten anderer Arten von irAEs ( 35\%). Da Schübe der Grunderkrankung in der Regel gut beherrscht werden können, stellt eine vorbestehende Autoimmunerkrankung keine Kontraindikation dar und sollte den Einsatz von ICl nicht ausschließen. Die Therapie rheumatischer irAEs richtet sich nach der Art der Manifestation und dem Schweregrad und sollte in enger Abstimmung des Rheumatologen mit dem Onkologen und dem Patienten erfolgen, wobei die ICI-Therapie bei Tumoransprechen wann immer möglich fortgesetzt werden sollte.
Der entscheidende Unterschied im Vergleich zu den klassischen Entitäten liegt darin, dass die Behandlung nicht das Treat-toTarget-Prinzip (mit dem Ziel der Remission) verfolgt, sondern in 1. Linie auf eine Beschwerdelinderung abzielt. Bei leichten bis moderaten muskuloskelettalen irAEs sind meist NSAR und/oder niedrig dosierte Glukokortikoide ausreichend, krankheitsmodifizierende Basistherapien wie Methotrexat oder gar Biologika kommen erst in 2. oder 3. Linie zur Glukokortikoid-Einsparung zum Einsatz. Schwere systemische irAEs wie Kollagenosen und Vaskulitiden werden, sofern Glukokortikoide nicht ausreichen, oft analog zu den klassischen Entitäten behandelt. Von der defensiven Therapiestrategie wird jedoch bei der $\mathrm{ICl}$-getriggerten Myositis (oft kombiniertes Auftreten mit Myokarditis oder Myasthenia gravis) aufgrund des häufig letalen Verlaufs insbesondere bei bulbärer Beteiligung abgewichen. Neben Absetzen des ICl sind hier regelhaft hochdosierte Glukokortikoide und Immunsuppressiva sowie ggf. intravenöse Immunglobuline oder Plasmapherese erforderlich.

Eine positive Nachricht für die Betroffenen ist, dass das Auftreten von irAEs im Allgemeinen und speziell auch von rheumatischen irAEs potenziell mit einem besseren Ansprechen auf die Tumortherapie assoziiert ist. Allerdings gehen rheumatische irAEs aufgrund des häufig chronischen Verlaufs oft mit hoher Morbidität einher. In diesem Kontext ist es eines der vordring lichsten Forschungsziele, die Behandlung von irAEs in den nächsten Jahren weiter zu optimieren, ohne dabei die antitumorale Wirkung der ICls zu beeinträchtigen. 